Pacific Journal of Mathematics

REPRESENTATION OF FINITELY GENERATED NILPOTENT 


\title{
REPRESENTATION OF FINITELY GENERATED NILPOTENT GROUPS
}

\author{
IAN D. BROWN
}

A. I. Malcev has shown that finitely generated torsion free nilpotent groups imbed as lattices in nilpotent Lie groups, and hence their structure is similar to that of the Lie groups. Since A. A. Kirillov has classified the representations of nilpotent Lie groups and, in particular, shown that they are all monomial (induced from one dimensional representations of subgroups), one might conjecture that representations of finitely generated nilpotent groups were monomial. (A representation, here, is a weakly continuous unitary representation on separable Hilbert space.) We prove a criterion for when a representations of finitely generated nilpotent groups are monomial. We will also show that representations induced from finite dimensional ones satisfy similar equivalence and irreducibility criteria to those deduced by Kirillov for nilpotent Lie groups.

To widen the conjecture slightly, one might ask about representations induced from finite dimensional representations. Our first lemma shows that this reduces to the question of monomial representations (using the theorem on inducing in stages).

Let us establish some notation. $\chi \uparrow G$ will be the representation of $G$ induced (definition in Mackey, [6]) from a representation $\chi$ of some subgroup. If $\chi$ is a character (one dimensional representation) on a subgroup $H$ of $G, \chi^{g}$ is the character on $H^{g}=g^{-1} H g$ given by $\chi^{g}(h)=\left(g h g^{-1}\right)$. If $U$ is a representation, $\mathscr{H}(U)$ is the space on which $U$ acts. Let $C$ be the complex numbers.

First of all, the wider conjecture reduces to the narrower via.

LEMMA 1. If $V$ is a finite dimensional irreducible representation of a nilpotent group $G$, then $V$ is induced from a one dimensional representation of some subgroup of $G$.

Proof. Consider $V$ as a representation of $G_{0}=G / \operatorname{Ker} V$. If $V$ is not one dimensional, $G_{0}$ is not abelian. Since $G_{0}$ is nilpotent, there is an abelian normal noncentral subgroup $A \subset G_{0}$. Then $V \mid A$ is a sum of characters. Call one of these $\chi$. Let $F=\left\{g \in G: \chi^{g}=\chi\right\}$. If $g \in G-F, g$ carries $\mathscr{H}(\chi)$, the subspace of $\mathscr{H}(V)$ on which $A$ acts according to $\chi$, onto $\mathscr{H}\left(\chi^{g}\right)$. Hence $\left[G_{0}: F\right]<\infty$. Since $F$ preserves $\mathscr{H}(\chi)$, we can let the representation of $F$ on $\mathscr{H}(\chi)$ be $U$. Now note that $F \neq G_{0}$ since $V$ is faithful and $A$ is not central hence not a 
multiple of a single character. Hence if we can prove $V$ is equivalent to $U \uparrow G_{0}$, we are done by induction on the dimension of $\mathscr{C}(V)$.

Let $f_{i}^{v} \in \mathscr{H}\left(U \uparrow G_{0}\right)$ be defined by $f_{i}^{v}\left(g_{k}\right)=\delta_{i k} v$ where $v \in \mathscr{H}(\chi)$, the $g_{k}$ are representatives for $G_{0} / F$ and $\delta_{i k}$ is the Kroneker $\delta$. Then the $f_{i}^{v} \operatorname{span} \mathscr{H}\left(U \uparrow G_{0}\right)$. Let $W f_{i}^{v}=V\left(g_{i}^{-1}\right) v$. Then $W: \mathscr{H}\left(U \uparrow G_{0}\right) \rightarrow$ $\mathscr{H}(V)$. One can check that $W$ commutes with the action of $G_{0}$ and is unitary, using the fact that $V\left(g_{i}\right) \mathscr{H}(\chi) \perp \mathscr{H}(\chi)$ for $g_{i} \neq e$. Thus we conclude that $V$ is induced as a representation of $G_{0}$, from a faithful representation of some subgroup of $G_{0}$, hence, by induction, from a character $\chi_{0}$ on some subgroup $H$ of $G_{0}$. Then, as a representation of $G, V$ is induced from $\chi_{0}^{\prime}$ on $p^{-1}(H)$ where $p: G \rightarrow G_{0}$ is the canonical projection and $\chi_{0}^{\prime}(g)=\chi_{0}(p(g))$.

Next, we need some information about finitely generated nilpotent groups.

Definitions. (1) If $N$ is a group, let $N^{i}=\left[N, N^{i-1}\right], N^{2}=[N, N]$ where $[A, B]$ is the subgroup generated by all commutators $a b a^{-1} b^{-1}$, $a \in A, b \in B$. (2) A nilpotent group is nilpotent of class $k$ if $N^{k+1}=1$, $N^{k} \neq 1$.

Lemma 2. A subgroup of a finitely generated nilpotent group is finitely generated.

Proof. Let $H$ be a subgroup of the finitely generated nilpotent group $G$. If $G$ is nilpotent of class $1, G$ is abelian and the result is quite easy.

Assume the result for all nilpotent groups of class $k$. If $G$ is nilpotent, of class $k+1, H \cap[G, G]$ is finitely generated as a subgroup of $[G, G]$. Also $H / H \cap[G, G]$, as a subgroup of $G /[G, G]$ is finitely generated since $G /[G, G]$ is abelian.

A set of representatives for the generators of $H / H \cap[G, G]$ plus the generators for $H \cap[G, G]$ will serve as a set of generators for $H$.

LEMMA 3. If $G$ is a finitely generated nilpotent group for any fixed $n$ there are only a finite number of subgroups of $G$ of index $n$.

Proof. The result is due to Howe [3] for a torsion free finitely generated nilpotent group. We show here how the result extends to any finitely generated nilpotent group.

Hirsch [2] has shown that $G$ is isomorphic to a subgroup of finite index in the direct product $A \times B$ where $A$ is a finite group and $B$ is a torsion free finitely generated nilpotent group. Then if $[G: H] \leqq r$ and $[A \times B: G]=n$, then $[A \times B: H] \leqq n+r$. Since $H / H \cap B \cong B H / B$ $[H: H \cap B]=[B H: B] \leqq|A|$. Thus $[A \times B: H \cap B] \leqq(n+r) \cdot|A|$ and 
$[B: H \cap B] \leqq n+r$. By Howe's result there are only a finite number of choices for $H \cap B$ and then, to complete the choice of $H$, one is left to select among less than $(n+r) \cdot|A|$ cosets of $H \cap B$ in $G$. Hence there are only a finite number of possibilities for $H$ given $r$ and $G$.

We now introduce, the

Definition. A subgroup $H$ of a group $G$ is called isolated if it contains all its roots, i.e., if $g^{n} \in H$ for some positive integer $n$, then $g \in H$.

We will denote the smallest isolated subgroup containing $H$ by $H^{*}$. Then $H^{*}$ has the following properties.

LEMMA 4.

(1) $H^{g^{*}}=H^{* g}$ for all $g \in G$

(2) $\left(H_{1} \cap H_{2}\right)^{*}=H_{1}^{*} \cap H_{2}^{*}$

(3) $N_{G}\left(H^{*}\right)=N_{G}(H)^{*}$

(4) $H / H^{g} \cap H$ and $H^{g} / H^{g} \cap H$ are finite $\Leftrightarrow g \in N_{G}\left(H^{*}\right)$.

Proof. (1) $k \in H^{g^{*}} \Leftrightarrow k^{n} \in H^{g}$ for some $n$

$$
\begin{aligned}
& \Leftrightarrow k n=g^{-1} h g \text { for some } n, h \\
& \Leftrightarrow\left(g k g^{-1}\right)^{n}=h \text { for some } n, h \\
& \Leftrightarrow g k g^{-1} \in H^{*} \\
& \Leftrightarrow k \in H^{* g}
\end{aligned}
$$

(2) $g \in\left(H_{1} \cap H_{2}\right)^{*} \Rightarrow g^{n} \in H_{1} \cap H_{2} \Rightarrow g \in H_{1}^{*} \cap H_{2}^{*}$

$$
\begin{aligned}
g \in H_{1}^{*} \cap H_{2}^{*} & \Rightarrow g^{n_{1}} \in H_{1} \text { and } g^{n_{2}} \in H_{2} \\
& \Rightarrow g^{n_{1} n_{2}} \in H_{1} \cap H_{2} \Rightarrow g \in\left(H_{1} \cap H_{2}\right)^{*}
\end{aligned}
$$

(3) $\left[H^{*}: H\right]$ is finite (from Baumslag [1], Lemma 2.8). If $g \in$ $N_{G}\left(H^{*}\right), g H g^{-1} \subset H^{*}$ of index $\left[H^{*}: H\right]$. Thus only a finite number of these $g H g^{-1}$ are different by Lemma 3. Thus $g^{n} \in N_{G}(H)$ for some $n$, i.e., $g \in N_{G}(H)^{*}$ and $N_{G}\left(H^{*}\right) \subset N_{G}(H)^{*}$. But $N_{G}\left(H^{*}\right)$ is isolated (Kuros [5], p. 249). Hence $N_{G}\left(H^{*}\right)^{*}=N_{G}\left(H^{*}\right)$. Also for $h \in H^{*} g \in N_{G}(H)$, if $h^{n} \in H, g h^{n} g^{-1}=\left(g h g^{-1}\right)^{n}$ is in $H$. Hence $g h g^{-1} \in H^{*}$ and $g \in N_{G}\left(H^{*}\right)$. Thus $N_{G}(H) \subset N_{G}\left(H^{*}\right)$, so we have

$$
N_{G}\left(H^{*}\right) \subset N_{G}(H)^{*} \subset N_{G}\left(H^{*}\right)^{*}=N_{G}\left(H^{*}\right) \text {. }
$$

Hence the result (3)

(4) $\left(H^{g} \cap H\right)^{*}=H^{* g} \cap H^{*} H / H^{g} \cap H$ is finite $\rightarrow H^{*} / H^{g} \cap H$ is finite $\Rightarrow\left(H^{g} \cap H\right)^{*}=H^{* g} \cap H^{*}=H^{*}$ (this because if $H \supset K$ are both isolated they may be considered as subgroups of torsion free $G /$ (torsion subgroup of $G$ ) and there it is easy to see that either $[H: K]=1$ or $[H: K]$ is infinite). Similarly $H^{g} / H^{g} \cap H$ finite $\Rightarrow H^{* g} \cap H^{*}=H^{* g}$. Hence the two finiteness conditions yield $g \in N_{G}\left(H^{*}\right)$. If $g \in N_{G}\left(H^{*}\right)$, 
$\left(H^{g} \cap H\right)^{*}=H^{*}$ and the two quotients are finite.

1. To develop our criterion mentioned above, we need a couple of lemmas, and for these we need the

DEFINITION. If $L, M$ are representations of a group $G$, and intertwining operator for $L$ and $M$ is a bounded linear operator $T: \mathscr{H}(L) \rightarrow$ $\mathscr{H}(M)$ such that $T L(g)=M(g) T$ for all $g \in G$.

The intertwining number, $i(L, M)$, of $L$ and $M$ is the dimension of the space of intertwining operators for $L$ and $M$.

The first lemma centres around a situation which will be the chief concern of this section. A subgroup $H$ preserves a one dimensional subspace of a certain representation space $\mathscr{H}(\rho)$. This action describes a character of $H$. If this character is induced to $G$, the lemma describes its relationship to the original representation $\rho$.

LEMMA 5. Let $\rho$ be a representation of $G, v \in \mathscr{H}(\rho)$ such that $\rho(H) v \subset C v$ where $H$ is some subgroup, and $\rho(g) v \perp v$ for all $g \in G-H$. Then if $\chi(h) v \equiv \rho(h) v, i(\chi \uparrow G, \rho)>0$.

Proof. We merely have to construct one nonzero intertwining operator.

Let $U=\chi \uparrow G, g_{i}$ be right coset representatives of $H$ and $f_{i}^{v}$ be in $\mathscr{H}(U)$ defined by $f_{i}^{v}\left(g_{j}\right)=v i=j$ and $f_{i}^{v}\left(g_{j}\right)=0 i \neq j$. Then $U(g) f_{i}^{v}=f_{k}^{w}$ where $w=\chi\left(g_{k} g g_{i}^{-1}\right) v$ and $g_{k}$ is the representative of $H g_{i} g^{-1}$. To see this,

$$
\begin{aligned}
U(g) f_{i}^{v}\left(h g_{j}\right) & =f_{i}^{v}\left(h g_{j} g\right) \\
& =\chi(h) f_{i}^{v}\left(g_{j} g\right) \\
& =\chi(h) f_{i}^{v}\left(g_{j} g g_{1}^{-1} g_{1}\right) \text { where } g_{1}
\end{aligned}
$$

represents

$$
H g_{j} g=\left\{\begin{array}{l}
01 \neq i \\
\chi(h) \chi\left(g_{k} g g_{i}^{-1}\right) v \quad 1=i \quad \text { since }
\end{array}\right.
$$

$1=i$ if and only if $g_{j} g \in H g_{i} \Leftrightarrow g_{j} \in H g_{i} g^{-1}$

$$
=\chi(h) f_{k}^{w}\left(g_{j}\right) \text { as claimed. Now define }
$$

$W: \mathscr{H}(U) \rightarrow \mathscr{H}(\rho)$ be

$$
W\left(f_{i}^{v}\right)=\rho\left(g_{i}^{-1}\right) v .
$$

Then one can show that $W U(g)=\rho(g) W$ for all $g \in G$, after defining $W$ on all of $\mathscr{H}(U)$ by linearity. Then if $W$ is bounded it is an intertwining operator. In fact, $W$ is an isometry. First note that for $f \in \mathscr{C}(U)$, 


$$
f=\sum f_{i}^{f\left(g_{i}\right)} \text {. }
$$

Thus

$$
\begin{aligned}
\left(W f, W f_{0}\right) & =\left(\sum \rho\left(g_{i}^{-1}\right) f\left(g_{i}\right), \Sigma \rho\left(g_{j}^{-1}\right) f_{0}\left(g_{j}\right)\right) \\
& =\sum_{i}\left(\rho\left(g_{i}^{-1}\right) f\left(g_{i}\right), \rho\left(g_{i}^{-1}\right) f_{0}\left(g_{i}\right)\right)
\end{aligned}
$$

by the perpendicularity hypothesis of the lemma,

$$
\begin{aligned}
& =\sum_{i}\left(f\left(g_{i}\right), f_{0}\left(g_{i}\right)\right) \\
& =\left(f, f_{0}\right) .
\end{aligned}
$$

The other lemma is concerned with the multiplicity of characters in the restriction of a representation.

LEMMA 6. If $\rho$ is a representation of a nilpotent group $H$ and $\chi$ is a one dimensional subrepresentation of $\rho$, and $H_{0}$ is a normal subgroup of $H$ such that $\left[H: H_{0}\right]$ is finite with the property that $\chi \mid H_{0}$ occurs an infinite number of times in $\rho \mid H_{0}\left(i . e ., i\left(\chi\left|H_{0}, \rho\right| H_{0}\right)\right.$ is infinite), then $\chi$ occurs an infinite number of times in $\rho$.

Proof. Choose an integer $k$ such that $H^{k} \subset H_{0}, H^{k-1} \not \subset H_{0}\left(H^{i}=\right.$ [H, $\left.\left.H^{i-1}\right]\right)$. Choose $h \in H^{k-1}-H_{0}$. Then the group $H_{1}$ generated by $h$ and $H_{0}$ is normal in $H$. Also, for some positive integer $n, h^{n} \in H_{0}$. Let $v_{i} \in \mathscr{H}(\rho)$ be an infinite independent set on which $H_{0}$ acts as $\chi \mid H_{0}$. Then the subspace spanned by the $\left\{h^{i} v_{r}: i=1, \cdots, n\right\}$ contains a vector on which $H_{1}$ acts as $\chi \mid H_{1}$. Namely, if $a=\chi(h)$ and

$$
w=\sum_{i=0}^{n-1} a^{n-i} \rho\left(h^{i}\right) v_{r}, \text { then }
$$

$h^{p} w=\chi(h)^{p} w$ for all integers $p$. Since there is an infinite number of independent $v_{r}$, there are an infinite number of independent such $w$ hence $\chi \mid H_{1}$ is of infinite multiplicity in $\rho \mid H_{1}$. Continuing this construction a finite number of times, we reach the conclusion of the lemma.

These two lemmas will be very useful in what follows. We now come to our criterion.

Definition. We will say a representation of a group $G$ has the finite multiplicity property if there is a subgroup $H$ which preserves a one dimensional subspace of $\mathscr{H}(\rho)$ with the additional property that if $\chi$ is the character of $H$ so defined, $\rho \mid H$ contains $\chi$ as a discrete direct summand only a finite number of times.

With this we can state. 
THEOREM 1. If $G$ is a finitely generated nilpotent group, an irreducible representation $\rho$ of $G$ has the finite multiplicity property if and only if $\rho$ is induced from a one dimensional representation of some subgroup of $G$.

Proof of Theorem 1. Assume $\rho$ has the finite multiplicity property.

Claim. There is a subgroup $H \subset G$ and a vector $v$ in $\mathscr{H}(\rho)$ such that $\rho(h) v=\chi(h) v$ for all $h \in H(\chi(h) \in C)$ with the additional property that $\chi \uparrow G$ is irreducible.

If this is so, $\chi \uparrow N_{G}\left(H^{*}\right)$ is irreducible, where $N_{G}\left(H^{*}\right)$ is the normalizer of $H^{*}$ in $G$. But this means, by Mackey [7], Theorem $3^{\prime}$, that $\chi^{g} \neq \chi$ on $H^{g} \cap H$ for any $g \in N_{G}\left(H^{*}\right)-H$. Choose $h \in H^{g} \cap H$ such that $\chi^{g}(h) \neq \chi(h)$. Then

$$
\begin{aligned}
(\rho(g) v, v) & =(\rho(h g) v, \rho(h) v) \\
& =\left(\rho(g) \chi^{g}(h) v, \chi(h) v\right) \\
& =\chi^{g}(h) \overline{\chi(h)}(\rho(g) v, v) .
\end{aligned}
$$

Since $\chi^{g}(h) \neq \chi(h)$, then $(\rho(g) v, v)=0$. Let $K=N_{G}\left(H^{*}\right)$. Then $\chi \uparrow K$ is an irreducible subrepresentation of the representation of $K$ given by the closed linear span of $\rho(K) v$, by Lemma 5 .

Let $N_{G}(K)=K_{1}$. Let $\chi \uparrow K=U$ and choose $g \in K_{1}-K$. Then by Mackey [7], Theorem $3^{\prime} U^{g}$ is not equivalent to $U$ since $U \uparrow G$ is irreducible.

Let $\mathscr{H}_{0}$ be a subspace of $\mathscr{H}(\rho)$ on which $K$ acts according to $U$. Then $g \mathscr{H}_{0} \perp \mathscr{H}_{0}$ for $g \in K_{1}-K$, since, if not, the projection of $g \mathscr{H}_{0}$ to $\mathscr{H}_{0}$ is an intertwining operator for $U^{g}$ and $U$.

By the form of $U$ there is a vector $v_{0} \in \mathscr{H}_{0}$ such that $\rho(k) v_{0} \perp v_{0}$ for all $k \in K-H$ and $\rho(h) v_{0}=\chi(h) v_{0}$ for all $h \in H$. Thus $\rho(g) v_{0} \perp v_{0}$ for all $g \in K_{1}-H$. Hence, by Lemma $5, \chi \uparrow K_{1}$ is a subrepresentation of the representation of $K_{1}$ on the closed linear span of $\rho\left(K_{1}\right) v_{0}$.

Since $G$ is nilpotent, a finite number of applications of this argument shows that $\chi \uparrow G$ is a subrepresentation of $\rho$. But $\rho$ is irreducible. Thus $\chi \uparrow G$ is equivalent to $\rho$.

Thus the "only if" part of the theorem is proven if we can establish the claim.

By Theorem $3^{\prime}$ of Mackey [7] we would be done if $\chi^{g} \neq \chi$ on $H^{g} \cap H$ for all $g \in N_{G}\left(H^{*}\right)$, where $H, \chi$ are as in the definition of the finite multiplicity property. We will produce a new triple $H^{\prime}, \chi^{\prime}, v^{\prime}$ with this property.

The first step is to satisfy this requirement for $g \in N_{G}\left(H^{*}\right)-H^{*}$. Let $H_{0}=\bigcap_{\left.g \in N_{G^{(H}}\right)} g^{-1} H g$. By (3) of Lemma 4 this intersection is finite. Hence, by (2) and (1) of Lemma $4, H_{0}^{*}=H^{*}$. Thus $\left[H: H_{0}\right]$ is finite. 
Let $\chi_{0}=\chi \mid H_{0}$ and $L=\left\{g \in N_{G}\left(H^{*}\right): \chi_{0}^{g}=\chi_{0}\right\}$. Then, using Lemma 6, the dimension of the space spanned by $L v$ is finite, since $\rho \mid H_{0}$ acts as $\chi_{0}$ thereon.

Then $\rho(L)$ on this subspace is a nilpotent unitary subgroup of $G L(n, C)$ for some $n$. Hence the connected component of its algebraic closure is diagonalizable. Thus $L$ has a subgroup $L_{0}$ such that [ $L: L_{0}$ ] is finite and there is a $v_{0}$ in the span of $\rho(L) v$ such that $t v_{0} \in C v_{0}$ for all $t \in L_{0}$. Now if there is a $g \in N_{G}\left(H^{*}\right)-H^{*}$ such that $\chi^{g}=\chi$ on $H^{g} \cap H$, then $\chi^{g}=\chi$ on $H_{0}$ and $g \in L$.

Let t.f. rank $K=$ torsion free rank $K=$ dimension of the simply connected nilpotent Lie group in which $K^{*} /($ torsion subgroup of $K$ ) imbeds as a uniform subgroup.

Then t.f. $\operatorname{rank} L>$ t.f. $\operatorname{rank} H$ and t.f. $\operatorname{rank} L_{0}>$ t.f. $\operatorname{rank} H$. Since $G /$ (torsion subgroup of $G$ ) imbeds as a uniform subgroup of a simply connected nilpotent Lie group, the torsion free ranks of the subgroups are bounded, so we can begin with an $H$ with the required properties and maximum rank, and the above argument shows that there is no $g \in N_{G}\left(H^{*}\right)-H^{*}$ such that $\chi^{g}=\chi$ on $H^{g} \cap H$. In fact, our argument is stronger-there is no $g \in N_{G}\left(H^{*}\right)-H^{*}$ such that $\chi^{g}=\chi$ on $H_{0}$.

Now let $L=\left\{g \in H^{*}: \chi^{g}\left|H_{0}=\chi\right| H_{0}\right\}$. Let $L^{i}=\left[L, L^{i-1}\right], L^{m} \subset H_{0}$ and $L^{m-1} \not \subset H_{0}$. Choose $g \in L^{m-1}-H_{0}$ and let $H_{1}$ be the group generated by $g$ and $H_{0}$. Then $H_{1}$ is normal in $L$ since $H_{0}$ is. Since $L \subset$ $H^{*}, g^{k} \in H_{0}$ for some positive integer $k$. Let $a$ be a $k$ th root of $\chi\left(g^{k}\right)$ and define $w$ as in the proof of Lemma 6. Then $\rho(g) w=a w$ and $\rho(h) w=\chi(h) w$ for all $h \in H_{0}$. Thus $\chi$ extends to a character $\chi_{1}$ of $H_{1}$. Let $L_{1}=\left\{t \in L: \chi_{1}^{t}=\chi_{1}\right\}$ and continue this process. Since $\left[H^{*}: H_{0}\right]$ is finite, this terminates with $L_{k}=H_{k}$, say.

Then for $g \in H^{*}-L_{k}, \chi^{g} \neq \chi$ on $H_{0}$ if $g \in H^{*}-L, \chi_{1}^{g} \neq \chi_{1}$ on $H_{1} \supset H_{0}$ if $g \in L-L_{1}, \cdots, \chi_{k}^{g} \neq \chi_{k}$ on $H_{k} \supset H_{k-1}$ if $g \in L_{k-1}-L_{k}$. Thus $\chi_{k}^{g} \neq \chi_{k}$ on $H_{k}$ for any $g \in H^{*}-L_{k}=H^{*}-H_{k}$. From the above paragraph, $\chi_{k}^{g} \neq \chi_{k}$ on $H_{0} \subset H_{k}$ for any $g \in N_{g}\left(H^{*}\right)-H^{*}$. Thus $\chi_{k}^{g} \neq$ $\chi_{k}$ on $H_{k}^{g} \cap H_{k}$ for $g \in N_{G}\left(H^{*}\right)-H_{k}$. Since $H^{*}=H_{k}^{*}$ this is precisely the subgroup-representation pair we were seeking. Hence we have the claim, and thus the "only if" part of the theorem.

Suppose $f \in \mathscr{H}(\chi \uparrow G)$ and $h f=\chi(h) f$. As in the proof of Lemma 2 , let $g_{i}$ be the right coset representatives of $H$. Let

$$
\begin{aligned}
f_{i}\left(g_{j}\right) & =1 & & i=j \\
& =0 & & i \neq j
\end{aligned}
$$

and define $f_{i}$ elsewhere so that it is in $\mathscr{H}(\chi \uparrow G)$. Then $f=\Sigma f\left(g_{i}\right) f_{i}$ and $h f_{i}=\chi\left(g_{k} h g_{i}^{-1}\right) f_{k}$ where $g_{k}$ is the representative of $H g_{i} h^{-1}$. Then 


$$
\begin{aligned}
h f & =\Sigma f\left(g_{i}\right) \chi\left(g_{k} h g_{i}^{-1}\right) f_{k} \\
& =\Sigma \chi(h) f\left(g_{s}\right) f_{s} .
\end{aligned}
$$

The $f_{i}$ are an orthonormal basis of $\mathscr{L}(\chi \uparrow G)$. Thus $\left|f\left(g_{i}\right)\right|=\left|f\left(g_{k}\right)\right|$ for all $\mathrm{h} \in H$. Thus for $f$ to be in $\mathscr{H}(\chi \uparrow G), H g_{i}$ must have a finite orbit in the cosets where $f\left(g_{i}\right) \neq 0$ for $f$ to have a finite norm. Thus there is a finite integer $n$ such that $g_{i} h^{n} \in H g_{i}$. i.e., $g_{i} h^{n} g_{i}^{-1} \in H$. Thus $g_{i} h g_{i}^{-1} \in H^{*}$ for each $h \in H$. Thus $g_{i} H g_{i}^{-1} \subset H^{*}$. Taking * on both sides, $g_{i} H^{*} g_{i}^{-1}=H^{*}$, hence $g_{i} \in N_{G}\left(H^{*}\right)$.

But $\chi \uparrow G$ is irreducible if and only if $i(\chi \uparrow G, \chi \uparrow G)=1$ and by Theorem 3 ' of Mackey [7], this is if and only if

$$
\sum_{\substack{d \in D_{f} \\ d \neq e}} i\left(\chi^{d}\left|H^{d} \cap H, \chi\right| H^{d} \cap H\right)=0 .
$$

Now $d \in D_{f}$ if and only if $d \in N_{G}\left(H^{*}\right)$. Hence for $d \in N_{G}\left(H^{*}\right)$ there is an $h \in H^{d} \cap H$ such that $\chi^{d}(h) \neq \chi(h)$. Now

$$
\begin{aligned}
h f(d) & =f(d h)=f\left(d h d^{-1} d\right) \\
& =\chi^{d}(h) f(d) \text { for } h \in H^{d} \cap H \\
& \neq \chi(h) f(d) \text { unless } f(d)=0 .
\end{aligned}
$$

Hence $f$ can only be nonzero on one right $H$ coset $-H$ itself. There it is determined once its value at $e$ is. Hence $\chi$ has multiplicity 1 in $\chi \uparrow G \mid H$.

2. Now let use this criterion to show there is representation of a nilpotent group which is not induced from any finite dimensional representation (or, equivalently, from any one-dimensional representation).

Our example will be a representation of the group of $3 \times 3$ matrices of the form

$$
\left[\begin{array}{lll}
1 & x & z \\
0 & 1 & y \\
0 & 0 & 1
\end{array}\right] \text { where } x, y, z \text { are integers }
$$

We will denote this matrix by $(x, y, z)$ so that

$$
(x, y, z)\left(x^{\prime}, y^{\prime}, z^{\prime}\right)=\left(x+y^{\prime}, y+y^{\prime}, z+z^{\prime}+x y^{\prime}\right) \text {. }
$$

First we describe a general form for a representation of $G$. A particular example of this will turn out to be the required counterexample. For $f$ in $L^{2}\left(S_{1}, \lambda\right)$ where $S_{1}$ is the circle and $\lambda$ is Lebesgue measure, let

$$
U_{s}(x, y, z) f(t)=e^{i(t y+b z)} s(x, t) f(t+x b) \text { where } b \text { is not in } Q \pi(Q \text { is }
$$
the rational numbers). This is a representation for any measurable 
$s: Z \times S_{1} \rightarrow S_{1}$, where the image circle is the unit circle of the complex numbers, if $s$ satisfies the property

$$
s\left(x+x^{\prime}, t\right)=s(x, t) s\left(x^{\prime}, t+x b\right)
$$

Note that, in fact, $s(1, t)$ can be an arbitrary measurable function, and then $s(x, t)$ is completely defined by $((1))$.

Let us now find out which $U_{s}$ are equivalent, and at the same time show that they are irreducible.

Lemma 7. The $U_{s}$ are irreducible and $U_{s_{1}}$ is equivalent to $U_{s_{2}}$ if and only if there is measurable $f: S_{1} \rightarrow S_{1}$ such that

$$
s_{1}(1, t)=f(t) \overline{f(t+b)} s_{2}(1, t) .
$$

Proof. Suppose $V: L^{2}\left(S_{1}, \lambda\right) \rightarrow L^{2}\left(S_{1}, \lambda\right)$ is unitary and $U_{s_{1}} V=$ $V U_{s_{2}}$ - i.e., $U_{s_{1}}(x, y, z) V=V U_{s_{2}}(x, y, z)$ for all integers $x, y, z$. Then

$$
V U_{s_{2}}(0, y, 0) f=U_{s_{1}}(0, y, 0) V f \text {. }
$$

Thus $V$ commutes with multiplication by $e^{i y t}$ for any integer $y$. Thus, by a known theorem of functional analysis (cf. Naimark, [10], p. 499, I) $V f(t)=v(t) f(t)$ for some measurable $v: S_{1} \rightarrow S_{1}$. Then $V U_{s_{2}}(x, 0,0)=$ $U_{s_{1}}(x, 0,0) V$ implies $v(t) s_{2}(x, t) f(t+x b)=s_{1}(x, t) v(t+x b) f(t+x b)$ for any $f$ in $L^{2}\left(S_{1}, \lambda\right)$ for all $t \in S_{1}$ and $x \in Z$. Thus

$$
v(t) s_{2}(x, t)=s_{1}(x, t) v(t+x b)
$$

is necessary for equivalence, and by the decomposition of $V$ above it can easily be shown to be sufficient. It is also easy to see that if $s(x, t)$ is constructed from $s(1, t)$ by $((1))$, then this equation is equivalent to $v(t) s_{2}(1, t)=s_{1}(1, t) v(t+b)$, as required. If $s_{1}=s_{2}$, then this means that $v(t)=v(t+b)-$ i.e. $v$ is invariant under translation by $b$. But this translation is an ergodic action on the circle. Hence, by a standard theorem of ergodic theory, $v$ is constant a.e., with respect to $\lambda$. Thus $V$ is in fact a scalar multiple of the identity operator, which shows that $U_{s}$ is irreducible.

Now let us look at a character $\chi$ and subgroup $H . U_{s}$ is infinite dimensional. Hence if $\chi \uparrow G$ is equivalent to $U_{s},[G: H]$ is infinite. Further, by Theorem 3' of Mackey [7]

$$
i(\chi \uparrow G, \chi \uparrow G)=\sum_{d \in D_{f}} i\left(\chi^{d}\left|H^{d} \cap H, \chi\right| H^{d} \cap H\right) .
$$

If $d=(0,0, z), d$ is central so that $H^{d}=H$ and $\chi^{d}=\chi$. Thus if $i(\chi \uparrow G, \chi \uparrow G)=1$, there can be only one $H: H$ double coset representative of the form $d=(0,0, z)$. Hence $(0,0, Z) \subset H$. From this 
and the fact that $[G: H]$ is infinite, we can see that $H=\{(n k, n p, z)$ : $n, z$ in $Z$ \}. Call this subgroup $H_{k p}$. If $\chi$ is a character on $H$, since $(0,0, Z)$ is central, $\chi((0,0, z))=e^{i w z}$ for some $w$ for all $z \in Z$. If $I$ is the identity operator, then $\chi \uparrow G((0,0, z))=e^{i w z} I$. But $U_{s}((0,0, z))=$ $e^{i b z}$. Hence $w=b$ if $\chi \uparrow G$ is equivalent to $U_{s}$.

Lemma 8. If $U_{s}$ is equivalent to $\chi \uparrow G$ for $\chi$ a character on $H_{k p}$, then $k=1$.

Proof. Let $K=H_{01}$. Parameterize the elements of $H_{k p}$ by $n$ and z. Let $[n, z]=(n k, n p, z)$. Then $[n, z]\left[n^{\prime}, z^{\prime}\right]=\left(n+n^{\prime}, z+z^{\prime}+n n^{\prime} k p\right)$. Then one can check that any character on $H_{k p}$ is of the form $\chi_{a b}$ where

$$
\chi_{a b}(n k, n p, z)=\exp i(n a-b n(n-1) k p / 2+b z) .
$$

First let us examine $\chi_{a b} \uparrow G \mid K$ where $\chi_{a b}$ is a character on $H_{k p}$ and $k \neq 0$. Then the $H_{k p}: K$ double coset representatives may be chosen $\{(x, 0,0): 0 \leqq x<k\}$. One can check that if $d=(x, 0,0), \chi_{a b}^{d}=\chi_{a+x p b, b}$. Then by Mackey's theorem (Mackey, [6], p. 117, Theorem 7.1)

$$
\begin{aligned}
\chi_{a b} \uparrow G \mid K & =\sum_{x=0}^{k-1}\left[\chi_{a b}^{d} \mid(0,0, Z)\right] \uparrow K \\
& =\sum_{x=0}^{k-1}\left[\chi_{0 b} \mid(0,0, Z)\right] \uparrow K \\
& =k
\end{aligned}
$$

copies of the regular representation of $(0, Z, 0)$ direct product with $\psi_{b}$ where $\psi_{b}(0,0, z)=e^{i b z}$. If $k \neq 1$, this has a commuting algebra which is not abelian. A projection onto one of the copies does not commute with the isomorphism between the copies. On the other hand,

$$
U_{s}(0, y, z) f(t)=e^{i(y t+b z)} f(t),
$$

which is precisely the regular representation of $(0, Z, 0)$ direct product with $\psi_{b}$. Using the theorem in Naimark ([10], p. 499, I), it is easy to see that this representation has an abelian commuting algebra. Hence to prove the result it remains to dispose of the case where $k=0$. Then $\chi_{a b} \uparrow G \mid K=\sum_{x=-\infty}^{\infty} \chi_{a b}^{d}$ by Mackey's theorem where $d=$ $(x, 0,0)$ if $p=1$. For $k=0$ and $p \neq 1$ or -1 it is not hard to show, using Theorem $3^{\prime}$ of Mackey [7], that $\chi_{a b} \uparrow G$ is not irreducible. Now if $U_{s}$ and $\chi_{a b} \uparrow G$ were equivalent for $\chi_{a b}$ defined on $H_{01}$, then $U_{s} \mid K$ and $\chi_{a b} \uparrow G \mid K$ would be equivalent, and by a theorem of Mackey (Mackey, [8], p. 103) Lebesgue measure would be measure isomorphic to the measure which weights each point of the orbit of $a$ as 1 . But this is a contradiction, since Lebesgue measure would then be atomic. 
This concludes the lemma.

Now some $U_{s}$ are equivalent to $\chi \uparrow G$ for some $\chi$. In fact we have the following lemma.

Lemma 9. If $\chi_{a b}$ is a character on $H_{1 p}, \chi_{a b} \uparrow G$ is equivalent to $U_{s}$ where $s(1, t)=e^{i(a-t p)}$.

Proof. For $f \in \mathscr{H}\left(\chi_{a b} \uparrow G\right)$ let $f^{\prime}(m)=f(0, m, 0)$. Then $f \rightarrow f^{\prime}$ is an isomorphism $\mathscr{H}\left(\chi_{a b} \uparrow G\right) \rightarrow l_{2}$ and

$$
\begin{aligned}
\chi_{a b} & \uparrow G(x, y, z) f((0, m, 0) \\
& =f(x, m+y, z) \\
& =f\left(\left(x, x p, z-x m-x y+x^{2} p\right)(0, m+y-x p, 0)\right) \\
& =\chi_{a b}\left(x, x p, z-x m-x y+x^{2} p\right) f^{\prime}(m+y-x p) .
\end{aligned}
$$

Taking Fourier transform,

$$
\begin{aligned}
\chi_{a b} \uparrow G(x, y, z) \hat{f}^{\prime}(t) \\
=\sum_{m} e^{-i t m} \chi_{a b} \uparrow G(x, y, z) f^{\prime}(m) \\
=\sum_{m} e^{-i t m} \chi_{a b}\left(x, x p, z-x m-x y+x^{2} p\right) f^{\prime}(m+y-x p) \\
=\sum \exp i[t(m-y+x p)-b x(m-y+x p)+x a-b x(x-1) p / 2 \\
\left.\quad+b\left(z-x y+x^{2} p\right)\right] f^{\prime}(m) \\
=e^{i(t y+b z)} \exp i[x a-b x(x-1) p / 2-t x p] \hat{f}^{\prime}(t+x b) .
\end{aligned}
$$

Thus comparing to the form of $U_{s}$,

$$
s(1, t)=e^{i(a-t p)} .
$$

CoROLLARY. $U_{s}$ is equivalent to a representation induced from a character on some subgroup if and only if there is some real a and integer $p$ and measurable $f: S_{1} \rightarrow S_{1}$ such that

$$
s(1, t)=e^{i(a-p t)} f(t) \overline{f(t+b)} .
$$

What we are going to show is that we can choose $s(1, t)$ so that this equation is impossible.

To do this, we need

LEMma 7. If $\chi_{a b}$ is a character on $H_{10}$ and $\chi_{u v}$ a character on $H_{1 p}$ for $p \neq 0$, then $\chi_{a b} \uparrow G$ is not equivalent to $\chi_{u v} \uparrow G$ for any choice of $a, b, u, v$. 
Proof.

$$
\begin{aligned}
& i\left(\chi_{a b} \uparrow G, \chi_{u v} \uparrow G\right) \\
& \quad=\sum_{d \varepsilon D f} i\left(\chi_{a b}^{d}\left|H_{10}^{d} \cap H_{1 p}, \chi_{u v}\right| H_{10}^{d} \cap H_{1 p}\right),
\end{aligned}
$$

by Mackey [7], Theorem 3'. But $H_{10}$ is normal and $H_{10} \cap H_{1 p}=(0,0, Z)$. Hence for no $d$ is $\left[H_{10}^{d}: H_{10}^{d} \cap H_{1 p}\right]$ finite. Thus $D_{f}$ is empty and the representations are not equivalent.

Let us note what this says according to the equivalence formula for the $U_{s}$. Substituting $s_{1}(1, t)=e^{i a}$ and $s_{2}(i, t)=e^{i(u-p t)}$ we find that

$$
e^{i a}=e^{i(u-p t)} f(t) \overline{f(t+b)}
$$

is false for any measurable $f: S_{1} \rightarrow S_{1}, p \neq 0$, and any $a, u$.

But by Theorem 1 , if $U_{s}$ is induced from $\chi$ there must be an $f \in \mathscr{H}\left(U_{s}\right)$ such that $U_{s}(h) f=\chi(h) f$ for all $h \in H$, the subgroup on which $\chi$ is defined. Thus there must be an $f \in L^{2}\left(S_{1}, \lambda\right)$ such that

$$
U_{s}(n, n p, z) f(t)=\exp i[n a-b n(n-1) p / 2+b z] f(t) \text {. }
$$

i.e.

$$
e^{i(n t p+b z)} s(n, t) f(t+n b)=\exp i[n a-b n(n-1) p / 2+b z] f(t) .
$$

Taking absolute values, this means $|f|$ is constant on orbits under translation by $b$. Hence $|f|$ is constant a.e., by ergodicity. Hence we can assume $f: S_{1} \rightarrow S_{1}$. Then for $n=1$, this says

$$
s(1, t)=e^{i a} f(t) \overline{f(t+b)} e^{-i t p} .
$$

$U_{s}$ is an irreducible representation if $s(1, t)=e^{i t / 2}$. Then

$$
e^{i t / 2}=e^{i a} e^{-i t p} f(t) \overline{f(t+b)}
$$

Then

$$
e^{i t(1+2 p)}=e^{i 2 a} f(t)^{2} \overline{f(t+b)^{2}} \text {. }
$$

Since $1+2 p \neq 0$ for any $p$, this contradicts (2). Hence this $U_{s}$ is not induced from a character.

3. Although every irreducible representation of a finitely generated nilpotent group is not induced from a character, it is useful to know exactly how to obtain those that are. In fact Mackey's Theorem $3^{\prime}$ of [7] leads quickly to a Kirillov type result. We have

THeOREM 2. Let $G$ be a finitely generated nilpotent group. Then

(1) If $H$ is a subgroup of $G, \chi$ a character (one dimensional representation) on $H$, and there is no $g \in N_{G}\left(H^{*}\right)-H$ such that $\chi^{g}=\chi$ on $H^{g} \cap H$, then $\chi \uparrow G$ is irreducible. 
(2) If $H$ is a subgroup of $G, V$ a finite dimensional representation $H$ such that $V \uparrow G$ is irreducible then $V \uparrow G$ is equivalent to $a$ representation obtained as in (1).

(3) If $\chi_{1}, \chi_{2}$ are characters on subgroups $H_{1}, H_{2}$ respectively satisfying the condition in (1), then $\chi_{1} \uparrow G$ is equivalent to $\chi_{2} \uparrow G$ if and only if there is a $g \in G$ such that

$$
H_{1}^{* g}=H_{2}^{*} \text { and } \chi_{1}^{g}=\chi_{2} \text { on } H_{1}^{g} \cap H_{2} \text {. }
$$

Proof. By the theorem of Mackey,

$$
i\left(\chi_{1}\left|G, \chi_{2}\right| G\right)=\sum_{d \in D_{f}} i\left(\chi_{1}^{d}\left|H_{1}^{d} \cap H_{2}, \chi_{2}\right| H_{1}^{d} \cap H_{2}\right)
$$

where $d \in D_{f}$ if and only if both $H_{1}^{d} / H_{1}^{d} \cap H_{2}$ and $H_{2} / H_{1}^{d} \cap H_{2}$ are finite

$$
\Leftrightarrow H_{1}^{d *} / H_{1}^{d} \cap H_{2} \text { and } H_{2}^{*} / H_{1}^{d} \cap H_{2}
$$

are finite

$$
\Leftrightarrow H_{1}^{d *} /\left(H_{1}^{d} \cap H_{2}\right)^{*} \text { and } H_{2}^{*} /\left(H_{1}^{d} \cap H_{2}\right)^{*}
$$

are finite

$$
\begin{aligned}
& \Leftrightarrow H_{1}^{d *}=H_{1}^{d *} \cap H_{2}^{*} \text { and } H_{2}^{*}=H_{1}^{d *} \cap H_{2}^{*} \\
& \Leftrightarrow H_{1}^{d *}=H_{1}^{* d}=H_{2}^{*} \text {. }
\end{aligned}
$$

From this (3) is immediate.

If we let $\chi_{1}=\chi_{2}=\chi$, then $d \in D_{f}$ if and only if $d \in N_{G}\left(H^{*}\right)$, and

(1) is immediate.

(2) is merely Lemma 1.

\section{REFERENCES}

1. G. Baumslag, Lecture Notes on Nilpotent Groups, Conference Board of Math. Sciences, Washington, 1971.

2. K. A. Hirsch, On infinite soluble groups III, Proc. London Math. Soc., (2) 49 (1946), 184-194.

3. R. Howe, On Representations of Discrete, Finitely Generated Torsion Free Nilpotent Groups, Preprint 1970.

4. A. A. Kirillov, Unitary representations of nilpotent Lie groups, Russian. Math. Survey, 17 No. 3, (1962), 53-104.

5. A. G. Kurosh, The of Groups, Vol. II, Chelsea, New York 1956.

6. G. W. Mackey, Induced representations of locally compact groups I, Ann. Math, 55 (2nd ser.) (1952), 101-139.

7. —, On induced representations of groups, Amer. J. Math., 73 (1951), 576-592.

8. - The Theory of Group Representations, Notes by Fell and Lowdenslager, Dept. of Math. of University of Chicago, 1955.

9. A. I. Malcev, On a class of homogeneous spaces, Amer. Math. Soc., Trans., Ser. 1, 9 (1962), 277-307.

10. M. A. Naimark, Normed Rings, 2nd. ed., P. Noordhoff N. V. Gronigen, 1964. 
Received November 17, 1970 and in revised form April 13, 1971 and revised again November 29, 1972. This article contains the main results of my Yale University doctoral dissertation. Many thanks are due to may supervisor G. D. Mostow for his unstinting encouragement and many helpful suggestions. This research was supported by a scholarship from Yale University.

UNIVERSITÉ DE RENNES

35-RENNES, FRANCE 


\section{PACIFIC JOURNAL OF MATHEMATICS}

EDITORS

\author{
H. SAMELSON \\ Stanford University \\ Stanford, California 94305 \\ C. R. HOBBY \\ University of Washington \\ Seattle, Washington 98105
}

\author{
J. DUGUNDJI \\ Department of Mathematics \\ University of Southern California \\ Los Angeles, California 90007 \\ RICHARD ARENS \\ University of California \\ Los Angeles, California 90024
}

\section{ASSOCIATE EDITORS}

E. F. BECKENBACH

B. H. NeUManN

F. WOLF

K. YosHIDA

\section{SUPPORTING INSTITUTIONS}

UNIVERSITY OF BRITISH COLUMBIA

CALIFORNIA INSTITUTE OF TECHNOLOGY

UNIVERSITY OF CALIFORNIA

MONTANA STATE UNIVERSITY

UNIVERSITY OF NEVADA

NEW MEXICO STATE UNIVERSITY

OREGON STATE UNIVERSITY

UNIVERSITY OF OREGON

OSAKA UNIVERSITY
UNIVERSITY OF SOUTHERN CALIFORNIA

STANFORD UNIVERSITY

UNIVERSITY OF TOKYO

UNIVERSITY OF UTAH

WASHINGTON STATE UNIVERSITY UNIVERSITY OF WASHINGTON

AMERICAN MATHEMATICAL SOCIETY NAVAL WEAPONS CENTER 


\section{Pacific Journal of Mathematics}

\section{Vol. 45, No. $1 \quad$ September, 1973}

William George Bade, Complementation problems for the Baire classes .......... 1

Ian Douglas Brown, Representation of finitely generated nilpotent groups ........ 13

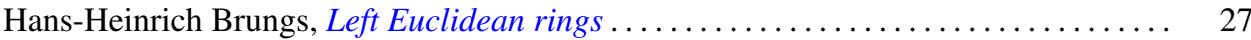

Victor P. Camillo and John Cozzens, A theorem on Noetherian hereditary rings ..... 35

James Cecil Cantrell, Codimension one embeddings of manifolds with locally flat

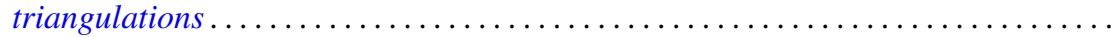

L. Carlitz, Enumeration of up-down permutations by number of rises . . . . . . . . . .

Thomas Ashland Chapman, Surgery and handle straightening in Hilbert cube

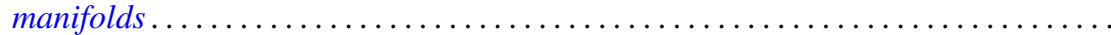

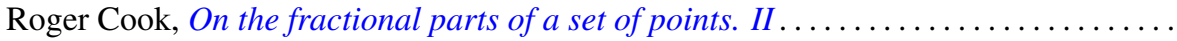

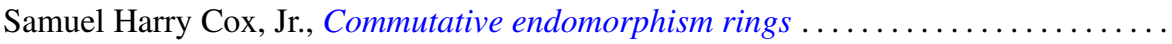

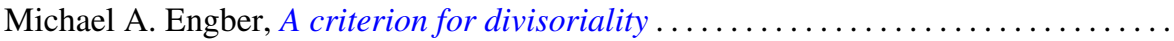

Carl Clifton Faith, When are proper cyclics injective . . . . . . . . . . . . . . 97

David Finkel, Local control and factorization of the focal subgroup . . . . . . . . . 113

Theodore William Gamelin and John Brady Garnett, Bounded approximation by

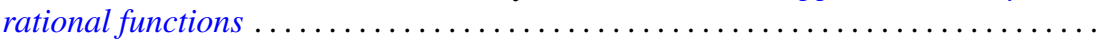

Kazimierz Goebel, On the minimal displacement of points under Lipschitzian

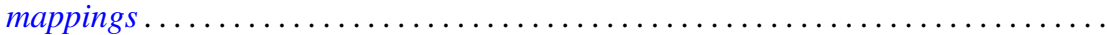

Frederick Paul Greenleaf and Martin Allen Moskowitz, Cyclic vectors for representations associated with positive definite measures: nonseparable

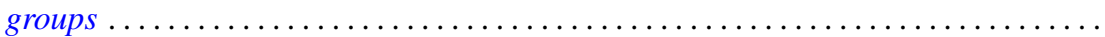

Thomas Guy Hallam and Nelson Onuchic, Asymptotic relations between perturbed linear systems of ordinary differential equations .

David Kent Harrison and Hoyt D. Warner, Infinite primes of fields and completions. .

James Michael Hornell, Divisorial complete intersections . ......

Jan W. Jaworowski, Equivariant extensions of maps ..............

John Jobe, Dendrites, dimension, and the inverse arc function .. .

Gerald William Johnson and David Lee Skoug, Feynman integrals of non-factorable

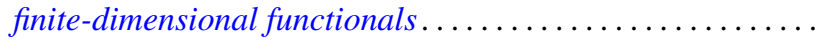

Dong S. Kim, A boundary for the algebras of bounded holomorphic functions ...... 269

Abel Klein, Renormalized products of the generalized free field and its derivatives ... 275

Joseph Michael Lambert, Simultaneous approximation and interpolation in $L_{1}$ and

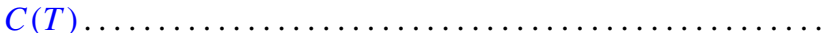

Kelly Denis McKennon, Multipliers of type $(p, p)$ and multipliers of the group

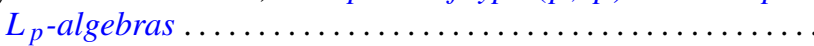

William Charles Nemitz and Thomas Paul Whaley, Varieties of implicative

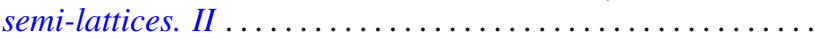

Donald Steven Passman, Some isolated subsets of infinite solvable

Norma Mary Piacun and Li Pi Su, Wallman compactifications on E-completely

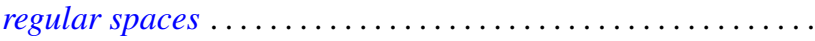

Jack Ray Porter and Charles I. Votaw, $S(\alpha)$ spaces and regular Hausdorff extensions....

Gary Sampson, Two-sided $L_{p}$ estimates of convolution transforms .

Ralph Edwin Showalter, Equations with operators forming a rig
Raymond Earl Smithson, Fixed points in partially ordered sets .

Victor Snaith and John James Ucci, Three remarks on symmetric products and

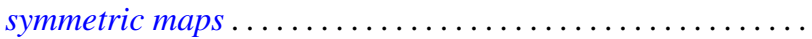

